nämlich in Form eines nach unten offenen Beutels ansgebildet, dessen Rand mit der flanschartig ausgestalteten unteren Randseite d einer entsprechenden Ausspalung in der anfgebogenen Einlegesohle a durch Kleben, Nähen oder dgl. fest verbunden und dessen untere offene Seite mittels einer der Sohle von unten her anliegenden und sie stützenden Metallplatte e hermetisch verschlossen ist.

Die bekannten Einlegesolılen fïr Plattrüßige haben vielfach den Nachteil, daß sie in der Hauptsache aus Metall bestehen, oder daß die für die Sohlenwölbung vorgesehene elastische Einlage den übrigen Stellen der Fußsoble sich nicht hinreichend anpassen läßt und daher hä口fig nach kurzem Gebrauch anfängt, den Benutzer zu belästigen. Diesen Uebelstand will D. R. P. 230880 (Ferdinand Schmeling in Erfurt) dadurch beseitigen, daß ein Bügelgestell aus Draht oder dgl. a (vgl. Fig. 12 bis 13) dem Umriß einer ganzen oder halben Sohle entsprechend geformt

Fig. 12

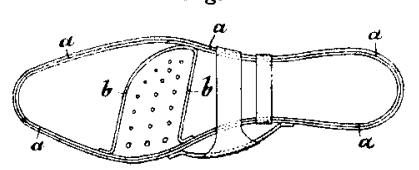

Fig. 13.

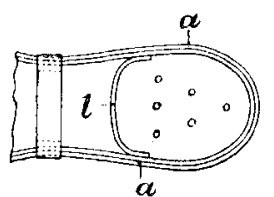

und von besonderen Quer- oder Verbindungsdrähten b, l der Uingienzung einzelner empfindlicher Fußstellen entsprechend durchizogen wird, woranf die in bekannter Weise aus Gumıni oder dgl. mit Lüftungslöchern hergestellten Einsatzstücke in die Zwischenräume eingelegt werden und mit flanschartigen, schon beim Gießen erzeugten Ansatzstücken auf den Metallumrandungen aufliegen.

Un eine Vorrichtung zur Ausfiihrung vou Operationen, insbesondere Gehirnoperationen, handelt es sich bei D. R. P. 230280 (Firma Wilhelm Holzhauer in Marburg). Die Vorrichtung besteht ans einem mit einer Vakuumleitung verbundenen, auf einem Gestell angeordneten

Kasten 1 (vgl. Fig. 14) mit einer in den Kasten einschiebbaren, zur Aufnahme des Patienten dienenden Opera. tionstischplatte und einer an der letzteren angelenkten, den

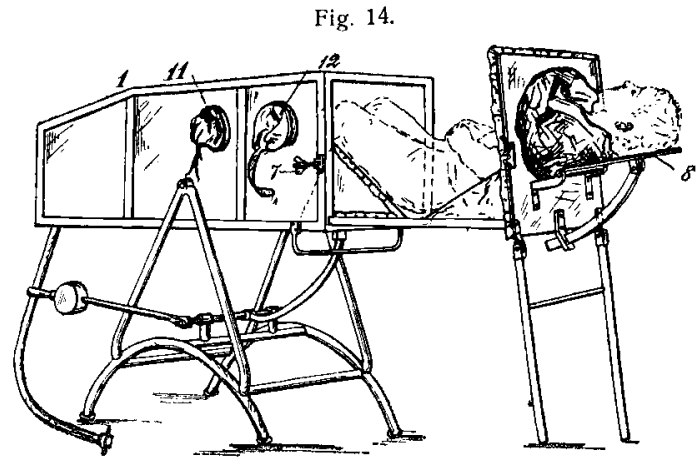
Kasten luftdicht

abschließenden Rückwand, welche in bekannter Weise mit einer durch eine Manschette 11, 12 abgedichteten Oeffnung zumn Hindurchstecken des Kopfes oder anderer Teile des Körpers versehen ist.

Die Fahrstiihle zur Selbstiortbewegung für Kranke, insbesondere an den Beinen gelähmter Personen gemäß den Patenten No. 217692 und 218137 (vgl. Deutsche medizinische Wochenschrift 1910, S. 942) haben durch das D. R. P. 230881 (Ed. Lange in Mühle-Siißenthal, Post Spiegelberg, Ostpr.) eine Verbesserung erfahren, inden der Vorderradträger 1 (vgl. Fig. 15 und 16) mittels einer lösbaren Zahnkupplıng p,

\section{Technische Neuheiten aus den Gebieten der Medizin, Oeffentlichen Gesundheitspflege und Krankenpflege.}

Von Dr. M. Schall in Halensee.

(Schlnß aus No. 22.)

Die mit Luitpolster zur Stiitzung des Fußgewölbes versehenen Eiılegesohlen haben durch D. R. P. 229989 (Cerf \& Bielschowsky in Frfurt) eine Verbesserung erfahren. Das Luftpolster c (vgl. Fig. 11) ist
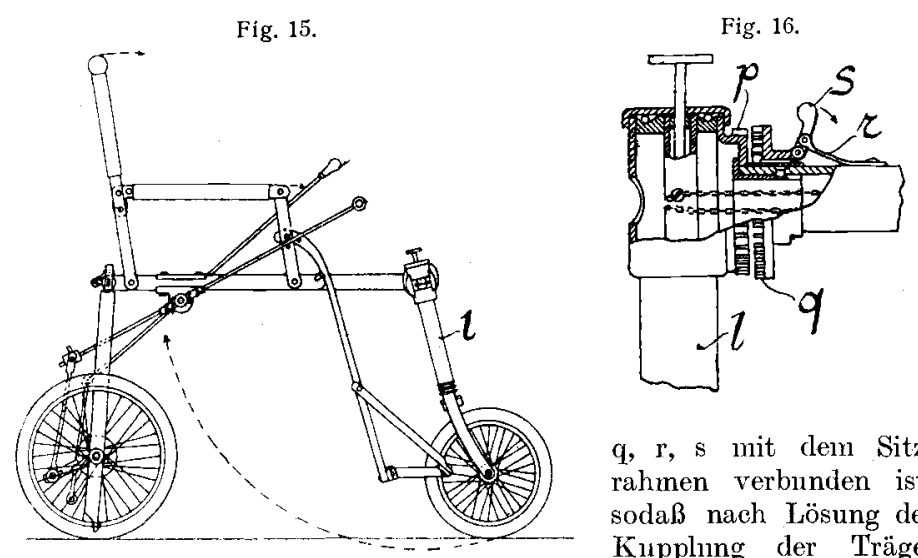

q, r, s mit dem Sitzrahmen verbunden ist, Kupplıng der Träger gegen den Sitz gelegt werden kann. Weiter besitzt der Frfindungsgegenstand den Vorteil, daß auch die Hinterräder verschiedene Winkelstellungen zum Sitzrahınen einnehmen können, wodurch der Fahrstuhl mehr oder weniger besodaß nach Lösung der schränkten Verkehrsverhältnissen sich leicht anpassen läßt. 


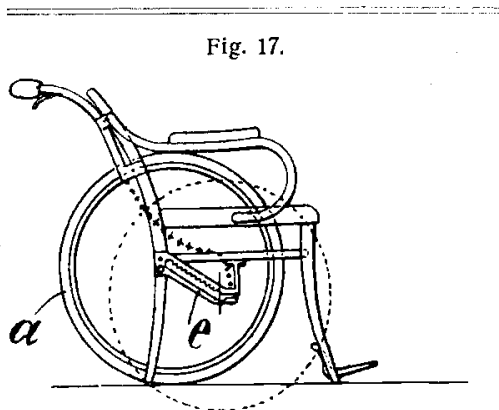

der letztere mit den Füßen vom Boden abgehoben wird.

Um eincn mechanisch-therapeutischen Apparat mit einem Elektromagneten, dessen Erregung durch einen mittels Schleifkontaktes einstell-

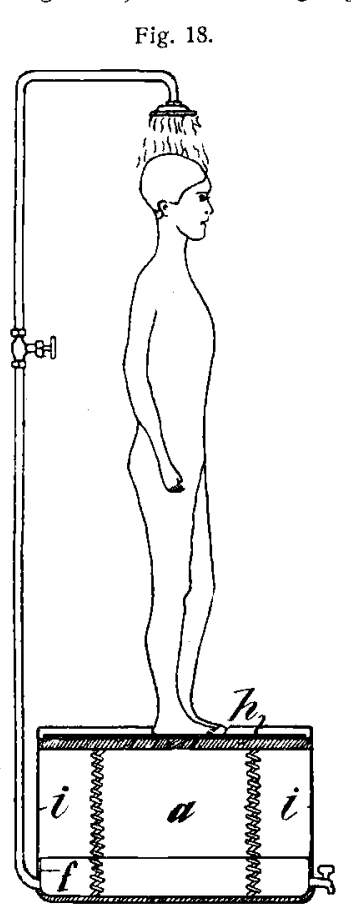
baren Widerstand geändert werden kann, handelt es sich bei D. R. F. 230504 (Oskar Helmer in Saint-Mandes, Seine, Frankr.). Der Schleifkontakt ist mit dem vom $\mathrm{Pa}$ tienten zu bedienenden Maschinenteil, z. B Welle oder Griff, verbunden, derart, daß der Schleifkontakt und damit der Widerstand entsprechend der Bewegung dieses Maschinenteiles verstellt wird.

D. R. P. 230197 (Jean Suchardt in Kassel) betrifft eine Duschvorrichtung, bei der das Wasser durch das Gewicht des Badenden emporgetrieben wird. Das kennzeichnende Merkmal der Erfindung besteht darin, daß die Fußplatte h (vgl. Fig. 18) unmittelbar mit der aus harmonikazugartig zusammenfaltbarem Stoff bestehenden Seitenwand i des Wasserbehälters a verbunden ist, sodaß eine Führung für die FuBplatte h nicht erforderlich ist.

Eine Klosettschutzvorrichtumg, bei der eine Papierbahn nach Münzeneinwurf selbsttätig über den Sitz gezogen wird, bildet den Gegenstand des D. R. P. 229928 (Ferd. Döring und Georg Arnold in Witzenhausen). Die das Papier aufwickelnde Automatenwelle e (vgl. Fig. 19) gibt bei ihrer Bewegung mit Hilfe eines Exzenters $f$ oder dgl. den Verschluß $m$ des sich selbsttätig öffnenden Klosettdeckels i frei.

Bei Verwendung von Papierstreifen mit bogenförmigen Ausschnitten als Schutzvorrichtung für Klosettsitze kann leicht ein Ein- oder Abreißen des abzuwickelnden Streifens eintreten. Um das zu vermeiden, wird gemä $B$ D. R. P. 229833 (Bèla Fuchs in Budapest) die Breite der Walzen so be messen, daß sie der Breite des Papierstreifens an den bogen. förmig ausgeschnittenen Stellen entspricht. Die den Papierstreifen abwickelnden Walzen sind dabei in bekannter Weise mit Mitteln versehen, durch die das benutzte Papier unbrauchbar gemacht wird.

D. R. P. 230577 (Dr. George Harker in Petershan bei Sydney, New-Süd-Wales, Australien) betrifft eine Vorrichtung zumDesin fizieren von Schiffen, Gebäuden usw. mit Mischungen von zerstäubten oder verdampiten brennbaren und explosionsiähigen Desinfektionsmitteln einerseits und unverbrennlichen Gasen anderseits. Das kennzeichnende Erfindungsmerkmal besteht in einem Verdampfer für flüssige, brennbare Desinfektionsmittel, der zusammen mit einem Mischer, Reiniger und Kühler für zugeführte Verbrennungsprodukte und Desinfektionsmittel so angeordnet ist, daß die gesamte Vorrichtung mit einem Druckmotor zum Einblasen der Gase in die Räume versehen, im AnschluB an eine vorhandene Feuerung oder Auspuff Verwendung findet.

Gemäß D. R. P. 230980 (Chemische Fabrik Flörsheim Dr. H. Nördlingen in Flörsheim a. M.) sollen formaldehydreiche Oel- und Fettmischungen für Desinfektionszwecke und dgl. hergestellt werden, indem säurehaltige Fette oder Oele tierischer oder pflanzlicher Herkunft, Harzöle oder freie Harz- oder Fettsäuren von fett- oder ölartigem Charakter mit Phenolen oder phenolreichen Teerölen einerseits, sowie mit Formaldehyd entweder gasförmig oder in wäßriger Lösung anderseits versetzt werden.

Gegenstand des D. R. P. 229971 (Firma Hausmann, A.-G., Schweizerisches Medizinal- und Sanitätsgeschäft in St. Gallen, Schweiz) ist eine Vorrichtung zum Desinfizieren der Spucknäpfe und des Sputums. Der Innenraum eines doppelwandigen, im Zwischenraum zur Dampferzeugung dienenden Kessels wird zwecks getrennter Desinfektion der Spucknäpfe und des Sputums mittels Dampf und Wasser in geeigneter Weise zweckmäßig durch einen mit der Schale nach unten gerichteten Trichter unterteilt. Nach Entfernung des Sputums ist der Desinfektor sofort wieder gebrauchsfertig.

Eine Strahldüse zum Befeuchten der Luit mit Dampf ist durch D. R. P. 230509 (Karl Ulrich in Zweibrücken, Pfalz) geschützt. Zu

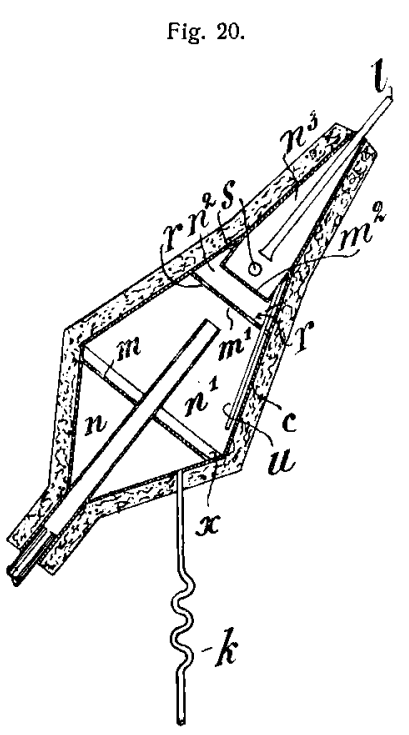
dem Zweck ist das Gehäuse c (vgl. Fig. 20) durch Zwischenwände $m$, $\mathrm{m}^{1}, \mathrm{~m}^{2}$ mit gegeneinander versetzten Löchern $r$, $s$ und mit unteren Durchlässen $u, x$ für das Tropfwasser zu einer gemeinsamen Ableitung $\mathbf{k}$ in einzelne Kammern $n, n^{1}, n^{2}, n^{3}$ geteilt und besitzt ein frei in dem Raum der letzten Kamner endendes Dampfaustrittsrohr l. Die Dampfzuleitung a liegt innerhalb der Tropfwasserableitung $\mathbf{b}$.

Gegenstand des D. R. P. 230694 Jules Henry Félix in Nogent-sur Varne, Seine, Frankreich) ist eine Luftpumpe mit Druckluftsammelisehälter fïr chirurgische $Z$ wecke, bei welcher der Pumpenkolben mit $\epsilon$ iner nach Art der Nürnberger Schere ausgebildeten Gelenkverbindung und einem Handhebel bewegt wird. Die Erfindung besteht darin, daß an der Gelenkverbindung eine auf eine Schiene sich stützende Zahnstange abgelenkt ist, in welche die Verzahnung eines mit dem Handhebel fest verbundenen Zahnsektors eingreift. Vorteilhaft wird die Gelenkverbindung und der Zahnstangenantrieb zwischen den tandomartig hinter-, bzw. übereinander angeordneten Pumpen und Iruckluft. sammelzylindern angeordnet. Der Gegenstand der Erfindung unterscheidet sich von bekannten Vorrichtungen ähnlicher Art dadurch, daß er einen verhältnismäßig sehr geringen Raum einnimmt. Die Antriebsvorrichtung ist so angeordnet, $\mathrm{da}$ man bei der Handhabung der Vorrichtung mit den Händen nicht in das Getriebe kommen kann und daß die Getriebeteile und die Ventile des Kompressors leicht zugänglich sind.

Durch D. R. P. 230741 (F. August Eggers in Hamburg) ist ein Bidet mit iestsitzender Unterdusche geschützt. Bei den bekannten Einrichtungen dieser Art kommt das Brausesieb mit dem bei Ausspülungen von Leibesöffinungen verunreinigten Waschwasser stets in unmittelbare Berührung. Dies ist in erhöhtem Maße dann der Fall, wenn das Bide vor Benutzung der Brause für Waschungen teilweise oder ganz angefüllt worden ist, und in der Praxis findet die Brause erst nach erfolgter Waschung Anwendung. Da aber das Waschwasser häufig noch für ärztliche Untersuchungen benötigt wird, so ist die Benutzung der Brause sofort nach geschehener Waschung unmöglich. Diese Uebelstände wer den durch den Gegenstand der vorliegenden Erfindung dadurch beseitigt, daß der Uebergang von dem den verschließbaren Ablaufstutzen (vgl. Fig. 21) aufnehmenden Bodenteil b zu dem den Brausekopf k aufnehmenden

Bodenteil c durch eine aufrechte Querwand hergestellt und gegenüber letz terer in der Seitenwand des $\mathrm{Bi}$ detbeckens eine Oeffnung $o$ in solcher Höhe an* geordnet ist, daß das ins Becken einfließende Wasser überlaufen und den

Brausekopf $\mathrm{k}$ nicht erreichen kann.

Um die Herstellung von Sauerstofibädern durch Katalyse von Superoxyden, Perboraten oder dgl. handelt es sich bei D. R. P. 230175 (L. Elkan Erben, G. m. b. H., in Berlin-Westend). Bei Sauerstoffbädern 
ist es von Vorteil, die Superoxyde, Perborate oder dgl. innerhalb der normalen Badedauer möglichst gleichmäßig und vollständig zu zersetzen, sowie einen möglichst hohen Grad von Uebersättigung des Badewassers mit Sauerstoff zu erzielen. In dieser Hinsicht verhalten sich die als Katalysatoren bekannten Substanzen ungemein verschieden. Es wurde nun gefunden, daß ein Zusatz von freiem Jod oder Jodalkalium die Wirksamkeit anderer, sei es anorganischer oder organischer Kataly. satoren erheblich steigert und außerdem sowohl die Entwicklung des Sauerstoffs regelt, als auch die Uebersättigung des Badewassers mit Sauerstoff begünstigt.

Ein Kehlkopfuntersuchungsapparat, bei dem die Lichtquelle mit dem Spiegel verbunden ist, der sich in der Verlängerung des Beobach-
Fig. 22.
tungstu-

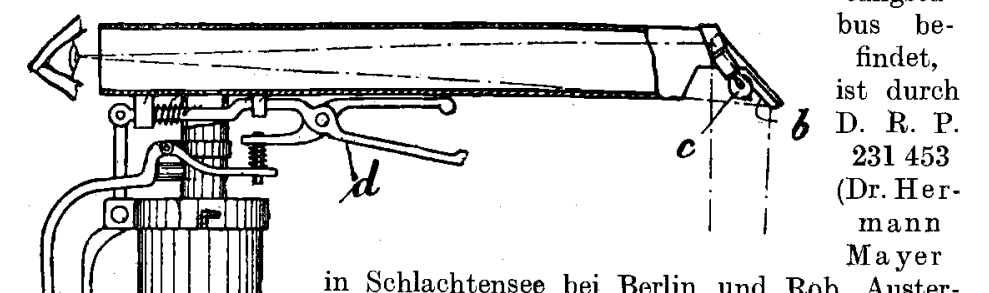

in Schlachtensee bei Berlin und Rob. Austerlitz in Berlin) geschützt. Der Erfindung gemäß wird die gleichmäßige Beleuchtung eines großen Gesichtsfeldes dadurch erreicht, daß seitlich und vorn neben dem Spiegel b (vgl. Fig. 22) zwei Lampen $\mathrm{c}$ vorgesehen sind. Ferner ist eine Zangeneinrichtung $d$ derart angeordnet, daß durch einen einzigen Hebeldruck die Zunge von der Zange erfaßt, aus der Rachenhöhle herausgezogen und gleichzeitig die Lampen des Apparates eingeschaltet werden.

Gegenstand des D. R. P. 231054 (Dr. Karl Lengfellner in Charlottenburg) ist ein Schulterstïtzapparat fiir Schlüsselbeinbruiche mit verstellbarer Rückenschiene und federnder Schulterstütze. Das Neue der Vorrichtung besteht darin, daß eine oder beide an der Rückenschiene 3 (vgl. Fig. 23) verstellbare Schulterstützen einerzeits an Schulterkappen 6, 7 angreifen, die durch regelbare, über Brust und Rücken geführte Gurte 8, 9 miteinander verspannt sind, anderseits mit einem von der Rückenschiene ausgehenden Halter einer federnden Druck. pelotte für die Bruchstelle verstellbar verbunden sind.

Die zur Erwärmung, besonders der Nieren dienende Heizvorrichtung nach D. R. P. 223219 (vgl. Deutsche medizinische Wochenschrift 1910 S. 2151) ist durch D. R. P. 231336 (Dr. Ernst Kuhn in Biebricha. Rh.)

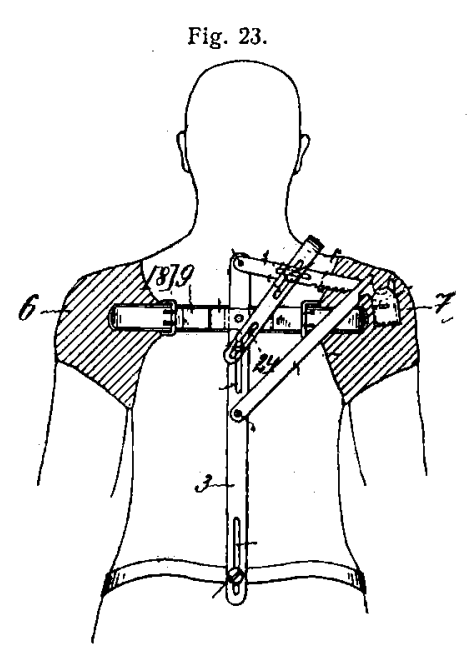
dadurch verbessert worden, daß an der den Beinen zugewandten Schmalkante des Wärmekastens ein oder zwei ansetzbare Nebenkästen vorgesehen sind, deren Auflagefläche der Form der betreffenden Gesäßhälfte und der Rückseite des anschließenden Oberschenkels angepaßt ist und deren Innenraum von demjenigen des Hauptkastens aus erwärmt werden kann.

D. R. P. 231288 (D. Gotthard Werner in Grafenberg bei Düsseldorf) schützt eine verstellbare Tragbahre mit einer Auflagefläche aus Tueh, einem Netz oder dgl., sowie einem aus Längs- und Querrohren oder -stäben gebildeten Grundrahmen. Auf den Holmen des letzteren sind die Füße eines das Kopfende des Tuches, sowie eines zweiten, eine Schenkelstütze tragenden Gestelles mittels paarweise verbundener, verschiebbarer und beliebig feststellbarer Muffen befestigt. 